\title{
Modellings for belief change: Prioritization and entrenchment*
}

by

HANS ROTT

University of Konstanz

\section{Introduction}

\subsection{Representation of beliefs}

OUR MODEL of belief will be a simple one. A belief is represented by a sentence in some (regimented) language. Research done in Artificial Intelligence has recently lead to a revival of the logic of belief. It was felt that a clear distinction should be drawn between the explicit and the implicit beliefs of a reasoner [15, 19]. The former ones are those that the reasoner would assent to if asked and for which he has some kind of independent warrant. The latter ones are those that follow, by some specified logic, from the set of explicit beliefs.

We distinguish a belief base, the set of explicit beliefs, from a belief set. A belief set is closed under logical consequences, it is a theory in the logician's sense. In general, we conceive of belief sets as generated by belief bases. Let us say that $H$ is a belief base for the belief set $K$ if and only if $K$ is the set of all logical consequences of $H$, i.e., if $K=C n(H)$.

We must make a decision what to count as a belief state. A belief state is that kind of thing, pre-theoretically understood, which is changed when we change our beliefs. As we cannot read off from a belief set $K$ which beliefs in it are the explicit ones, a belief state cannot be just a belief set. Should we say that a belief state is modelled by a belief base $H$ ? Of course, we then have no problem in generating the full belief set, provided we have fixed an

\footnotetext{
* I should like to thank Ulf Friedrichsdorf, Sven Ove Hansson, David Pearce, and an anonymous referee for clarifying discussions and comments. A preliminary version of this paper reporting on the very special case of belief bases without prioritization appeared under the title 'Modellings for belief change: Base contraction, multiple contraction, and epistemic entrenchment (Preliminary report)' in the Proceedings JELIA '92, 3rd European Workshop on Logics in AI, Berlin, September 7-10, 1992, Springer LNAI series 633, pp. 139-153.
} 
appropriate logic $C n$. However, as we shall see, there is a dynamical problem with this conception. In the sort of changes we shall consider, we cannot satisfy two desiderata at the same time: the desideratum that the changed belief state can be characterized by a belief base, and the desideratum that this belief base contains the set of explicit beliefs after the change has been effected. This is an unpleasant state of affairs which we shall have to put up with in this paper. Giving an answer to our question, we say that a belief state is a pair $\langle H, K\rangle$ such that $H$ is a belief base for $K$. However, the reader be warned that our change operations are not making belief states out of belief states in response to a certain input. We shall explain this in the next section.

Before doing that, let us delineate the object language and its logic. The logic of belief change, and especially the theory of epistemic entrenchment, has been discussed for a language with the expressiveness of propositional logic, including all its connectives $\neg, \wedge, \vee, \rightarrow$ and $\leftrightarrow$, as well as the truth and falsity constants $T$ and $\perp$. In contrast to this, we will aim at reducing the linguistic prerequisites. Our considerations are to apply also to systems using severely restricted languages, as encountered e.g. in inheritance nets or truth (reason) maintenance systems.

Correspondingly, the logic governing our language has to obey only structural rules. We require that it be reflexive, monotonic, transitive, and compact. We refer to our logic either as a consequence operator $C n$ or as an inference relation $\vdash$, with the usual understanding that $\phi \in C n(H)$ iff $H \vdash \phi$. In the first notation our four requirements become

(R) $H \subseteq C n(H)$

(M) If $H \subseteq H^{\prime}$ then $C n(H) \subseteq C n\left(H^{\prime}\right)$

(T) $C n(C n(H)) \subseteq C n(H)$

(C) If $\phi \in C n(H)$ then $\phi \in C n(H)$ for some finite subset $H^{\prime}$ of $H$

When linking our considerations to earlier work, we shall make use of connectives. Then the logic is further supposed to be supraclassical, i.e., what follows classically from a given premise set should follow from it in $C n$. We also assume that $C n$ satisfies the deduction theorem. 


\subsection{Dynamics}

A belief change occurs if a belief state is changed in order to accommodate it to a certain input. In the case we are going to deal with, the input comes in the form of (explicit) beliefs. In the research program initiated by Alchourrón, Gärdenfors and Makinson ([3]; for excellent surveys, see [7] and [17]), belief states are identified with belief sets, and inputs are single sentences. Still working in broadly the same research program, Fuhrmann $[5,6]$ and Hansson $[10,11,12]$ offer modellings for two important generalizations. They investigate what happens when belief states are modelled as belief bases (with belief sets as special cases) and when the input comes in sets of sentences (with singletons as special cases). In short, they generalize the theory of belief change to base changes and multiple changes.

It is clear from the very beginning that the idea of base change is indeed compelling. True, it is reasonable to say that what an agent really believes is the belief set $K$, including the full set of his implicit beliefs. But it is at least as reasonable to think of belief change operations as acting on the set of explicit beliefs alone. After all, merely implicit beliefs have a secondary status, they are derived from the explicit ones. And if some of the explicit beliefs they depend on should have to give way, so should they! What counts in belief revision is the explicit beliefs, and later we shall provide for the possibility that they count in varying degrees. This is a foundationalist picture of belief revision and contrasts with the coherentist picture predominant in the current theory of belief revision $[8,14]$. We will endorse the philosophy of base change in this paper.

Again, it is a good idea to be ready for set-like inputs. But this issue does not seem to have the same philosophical force as base contraction. Philosophically, base change is an alternative to theory change, while multiple change is just an extension of singleton change. There seems to be no intimate connection between these two kinds of deviation from the original framework of Alchourrón, Gärdenfors and Makinson. However, we shall argue that multiple belief changes play a significant role in the analysis of base changes. 


\subsection{Three types of belief change}

The simplest type of belief change is the addition of a new belief $\phi$ (or a set of beliefs) which is consistent with the old beliefs. In this case, we have no problem in identifying the relevant operations. We can effect theory change through base change. Using the symbol ' + ', we define consistent additions as follows:

$$
\begin{gathered}
H+\phi=H \cup\{\phi\} \\
K+\phi=C n(K \cup\{\phi\})
\end{gathered}
$$

Notice that ' + ' has two different meanings here, depending on whether its first argument is supposed to be a belief base or a belief set. It is obvious how to generalize these definitions when the input comes in sets. However, as the generalization will be far from obvious in the remaining cases, we shall restrict ourselves to singleton inputs in the rest of this section.

The operation of accommodating a belief state to some input is considerably more difficult if the latter is inconsistent with the former. In this case, it is usually held that consistency should act as an integrity constraint for our belief system. For such beliefcontravening additions, we shall adopt the following idea: In order to rationally include $\phi$ into the set $H$ (or $K$ ) of your beliefs, first make $H$ (or $K$ ) consistent with $\phi$, i.e., recant the commitment to $\neg \phi$, and then add $\phi$ consistently to the resulting set. It is common to use the term 'revision' to cover both consistent and belief-contravening additions, and to use the symbols ' $*$ ' for revisions and '-' for contractions. The above idea which is credited to Isaac Levi in the literature then becomes:

$$
\begin{gathered}
H * \phi=(H-\neg \phi)+\phi=(H \dot{-} \neg \phi) \cup\{\phi\} \\
K * \phi=(K-\neg \phi)+\phi=C n((K \dot{-} \neg \phi) \cup\{\phi\})
\end{gathered}
$$

This is the Levi identity, in its two versions for base and for theory change. One may think that the Levi identity is not of much help as long as we do not know how the contraction operation - behaves. This is right, but still it reduces the problem of finding suitable revision operations to the problem of finding suit- 
able contraction operations. Philosophically, contraction appears to be the more fundamental operation. Like most authors in belief revision, we shall follow Levi's advice and concentrate on the study of belief contractions in the following.'

What is this fundamental interesting operation called 'contraction'? The contraction of a set of beliefs with respect to an input sentence $\phi$ is a subset of the original beliefs which does not logically imply $\phi$. (In a sense, "input sentences" for contractions are rather "output sentences".) In case we start with a belief set $K$, we should end up with another belief set $K \dot{-} \phi$ which is logically closed again. In contrast to the case of additions, we do not want to stipulate that the contracted belief set $K \dot{-} \phi$ can always be identified with the set of logical consequences of a new belief base $H \dot{-} \phi$. We will explain why presently.

\subsection{The basic idea of minimal change}

When forced to perform a belief change, it seems a rational maxim to preserve as many of the most important beliefs as possible. Many writers have embraced such a condition of minimal change (minimum mutilation, maximal conservativity, informational economy) for many different purposes [18]. We will use the label 'minimal change approach' as a proper name for an account of belief revision which covers at least maxichoice, full, and partial meet contraction in the sense of Alchourrón, Gärdenfors and Makinson [3].

This is the basic idea of minimal change: In order to contract a belief base $H$ (or a belief set $K$ ) with respect to $\phi$, look at the maximal subsets of $H$ (of $K$ ) which do not imply $\phi$. Since every piece of information is valuable, no gratuitous loss of beliefs is tolerated. As a first shot, then, we say that a set $H_{1}$ of beliefs is better than, or preferred to, a set $H_{2}$, relative to the belief base $H$, if $H_{1}$ preserves more explicit beliefs than $H_{2}$, that is, if $H_{2} \cap H$ is a proper subset of $H_{1} \cap H$. In this case we write $H_{2} \prec H_{1}$. If $H_{1}$ and $H_{2}$ are subsets of $H$, this of course reduces to $H_{2} \subset H_{1}$. Proper inclusion is

"However, it is not easy to formulate analogues of the Levi identity for "pick" and "bunch revisions" in the sense explained below. 
a crude preference relation for sets of beliefs, but it is the bestbecause the only-thing we can do, as long as the syntactical structure of the belief base is supposed to be the only information available for belief change. We write $H \Delta \phi$ for the set of all $\prec-$ maximal or "best" subsets of $H$ which fail to imply $\phi$. As we shall presently consider more sophisticated preference relations $\prec$, we occasionally use Alchourrón and Makinson's [1] notation $H \perp \phi$ rather than $H \Delta \phi$ in order to mark the cases where $\prec$ is supposed to be fixed as proper inclusion (relativized to $H$ ).

\subsection{Base contraction}

In the following, the term 'base contraction' is not to be taken literally. What is changed is the theory $K=C n(H)$ generated by a base $H$. But how the theory is changed depends on the way it is axiomatized, on the structure of $H$. For instance, while $H=\{p, q\}$ and $H^{\prime}=\{p \wedge q\}$ generate the same theory $K$, we expect that $K \dot{-} p$ contains $q$ if $K$ is axiomatized by $H$, but that $q$ is lost if $K$ is axiomatized by $H^{\prime}$. In the latter case, $q$ is inseparable from $p$.

The minimal change approach is afflicted with a decisive difficulty. In general, there is more than one solution to the minimal change problem, i.e., more than one maximal set of beliefs which does not imply $\phi$. The point is that there is usually more than just one member in $H \Delta \phi$. What then to do? We adopt an egalitarians's point of view. All elements of $H \Delta \phi$ are to be treated equally.

The bold or credulous option is maxichoice base change: In order to eliminate $\phi$ from $K$, choose one element of $H \Delta \phi$ at random, and close under $C n$.

DEFINITION 1. Let $H$ be a base for $K$ and $\gamma$ be a (single-valued) choice function which selects, for every nonempty set $H \Delta \phi$, an arbitrary element of $H \Delta \phi$. Then the maxichoice base contraction over $K$ determined by $H$ and $\gamma$ is given by

$$
\psi \in K \dot{-} \phi \text { iff } \quad H \phi \text { and } \gamma(H \Delta \phi) \vdash \psi \text {, or } \vdash \phi \text { and } \psi \in K \text {. }
$$

Being maximally conservative, maxichoice contraction comes as 
close to the idea of minimal change as possible. However, if we do not have any tie-breaking information to govern the choice of some particular element of $H \Delta \phi$, there is no guarantee that the randomizing function $\gamma$ selects "the right" one. Believers do not play dice. The arbitrariness of maxichoice contractions is avoided by the next model for belief revision.

The skeptical option is meet base change: In order to eliminate $\phi$ from $K$, take all the elements of $H \Delta \phi$, then close each under $C n$, and finally take the intersection. ${ }^{2}$

Definition 2. For any base $H$ for $K$, the meet contraction over $K$ determined by $H$ is given by

$$
\begin{gathered}
\psi \in K-\phi \text { iff } H \phi \text { and } H^{\prime} \vdash \psi \text { for every } H^{\prime} \in H \Delta \phi, \\
\text { or } \vdash \phi \text { and } \psi \in K .
\end{gathered}
$$

Meet contractions depart from the idea of minimal change, because the intersection of several maximal non-implying subsets under $\prec$ is not itself a maximal non-implying subset under $\prec$. However, a symmetrical consideration of each element of $H \Delta \phi$ is required by our decision to let in no other information than is encoded in the structure of the explicit beliefs. Opting for meet contraction thus means adhering to the equality of rights of the members in $H \Delta \phi$.

Alchourrón and Makinson [1] have shown that meet contractions make good sense only if either $\prec$ allows for finer discriminations than $C$ or $H$ is a non-theory. If we have only syntactical information at our disposal, i.e., if every datum in the belief base carries the same epistemic weight, then it is essential for the following constructions that we have a differentiation between explicit and implicit beliefs. In this case the distinction is not only desirable intuitively, but also a technical prerequisite.

It would not be quite right to characterize our proposals as

\footnotetext{
2 Specializing $\prec$ to $\subset$, we get a method which is applied by Veltman [29] and Kratzer [13] for the analysis of counterfactuals, and by Poole [22] for noumonotonic reasoning. For more connections with the latter area of research, see Nebel [21].
} 
"theory change through base change" [6]. We do not want to stipulate that $K \dot{-} \phi=C n(H \dot{-} \phi)$ for some appropriate $H \dot{-} \phi$. Let us illustrate why. Consider $H=\{p, q\}$ and retract $p \wedge q$ from $K=C n(H)$. Then $H \perp(p \wedge q)=\{\{p\},\{q\}\}$, so under $K \dot{-} \phi=C n(H-\phi)$, maxichoice would give us either $K \dot{-}(p \wedge q)=C n(\{p\})$ or $K \dot{-}(p \wedge q)$ $=C n(\{q\})$, while meet would give us $K \dot{-}(p \wedge q)=C n(\emptyset)$. Neither of these solutions seems satisfactory. Intuitively, $K \dot{-}(p \wedge q)=$ $C n(\{p \vee q\})$ would be good. Even after conceding that one of $p$ and $q$ may be false, we should still cling to the belief that the other one is true. But $H^{\prime}=\{p \vee q\}$ is no base which can be constructed naturally from $H$-it certainly does not record any explicit belief. We are faced with a deep-seated dilemma. Either we must give up the philosophy of base changes which identifies the elements of a belief base with explicit beliefs, or else violate the methodological principle of categorial matching which requires that the result of a belief change should be of the same format as the initial representation of our beliefs. ${ }^{3}$ In this paper we are going to stick to the interpretation of bases as explicit beliefs. With a heavy heart, we forgo the aim of getting $\langle K \dot{-} \phi, H \dot{-}\rangle\rangle$ from $\langle K, H\rangle$ and stay content with the more modest aim of getting $K \dot{-} \phi$ from $K$ with the help of the belief base $H$. We assume that the belief base $H$ and the ordering $\prec$ of its subsets induced by the idea of minimal change are relevant, and indeed all that is relevant, for the construction of $K \dot{-\phi}$ from $K$, but neither $H$ nor the ordering of its subsets will get revised itself. Pictorially, instead of the desirable transition $\langle K, H, \prec\rangle$ $\stackrel{\phi}{\mapsto}\left\langle K \dot{-} \phi, H \dot{-} \phi, \prec^{*}\right\rangle$ we will study the transition $K \stackrel{\phi H<}{\leftrightarrow} K \dot{-} \phi$. In particular, there will be no suggestion as to the contents of $H \dot{-\phi}$.

\subsection{Multiple contraction}

When the input comes in sets, we are presented with two different kinds of contraction. The task of a pick contraction is to discard at least one element of a set $S$, while the task of a bunch contraction is to discard each element of a set $S$, both times with minimal mutilation of the original belief state. ${ }^{4}$ In conformity with the

${ }^{3}$ This principle is necessary and sufficient for a modelling of iterated revisions. 
basic idea of minimal change, we again focus on maximal non-implying subsets of $H$.

Let $H \Delta\langle S\rangle$ be the set of all maximal subsets of $H$ under $\prec$ which do not imply every element of $S$, and $H \Delta[S]$ the set of all maximal subsets of $H$ under $\prec$ which do not imply any element of $S$. Clearly, $H \Delta\langle\{\phi\}\rangle=H \Delta[\{\phi\}]=H \Delta \phi$. We will drop curly brackets within pointed and square brackets, so $K \dot{-}[\{\phi, \psi\}]$ will simplify to $K-[\phi, \psi]$, and $H \Delta\langle\{\phi, \psi\}\rangle$ to $H \Delta\langle\phi, \psi\rangle$, etc.

Notice that $H \Delta\langle\phi, \psi\rangle=H \Delta(\phi \wedge \psi)$. But there are no interesting analogues for $H \Delta[\phi, \psi]$. It seems that pick contractions with respect to finite sets are reducible to contractions of conjunctions, but that bunch contractions cannot easily be reduced to singleton contractions. $^{5}$

The concepts of maxichoice and meet contraction determined by a belief base can be generalized naturally to cover pick and bunch contractions as well. As the case of maxichoice contractions is entirely analogous, we restrict ourselves to meet contractions. Borrowing Fuhrmann's [5] symbols, we introduce

Definition 3. For any base $H$ for $K$, the pick and bunch versions of multiple meet contraction over $K$ determined by $H$ are defined as follows:

\footnotetext{
4 André Fuhrmann [5] was probably the first to study pick and bunch contractions. He called them choice and meet contractions. For danger of confusion with maxichoice and (full, partial) meet contraction, we introduce new names.

${ }^{5}$ We note some special cases for $\prec=\subset$. For theories $K$ and sentences $\phi$ and $\psi$ such that at least one of $\phi$ and $\psi$ is in $K, K \perp(\phi \vee \psi) \subseteq K \perp[\phi, \psi]$. For the proof of this and related things, the following fact is relevant: For every theory $K$, every $K^{\prime} \in$ $K \perp \phi$ and every $\psi$ and $\chi$ in $K-K^{\prime}$, it holds that $\psi \leftrightarrow \chi \in K^{\prime}$. This in turn is due to the fact that for every such $K, K^{\prime}$ and $\psi$, it holds that (i) $\psi \rightarrow \phi \in K^{\prime}$ (Proof: $K^{\prime} \vdash \psi \rightarrow \phi$, by maximality, so $\psi \rightarrow \phi \in K^{\prime}$, by $\psi \rightarrow \phi \in K$ [theory!] and maximality) and (ii) $\phi \rightarrow \psi$ $\in K^{\prime}$ (Proof: since $K^{\prime} H \phi$, we get $K^{\prime} H(\phi \rightarrow \psi) \rightarrow \phi$, so $K^{\prime} \cup\{\phi \rightarrow \psi\} \forall \phi$, so $\phi \rightarrow \psi \in K^{\prime}$, by $\phi \rightarrow \psi \in K$ [theory!] and maximality). However, in general $K \perp[\phi, \psi] \subseteq K \perp(\phi \vee \psi)$ even if $K$ is a theory; consider $K=\operatorname{Cn}(\{p, q\})$, for which $K \perp[p, q]=\{\operatorname{Cn}(p \vee q)$, $C n(p \leftrightarrow q)\}$, but $K \perp(p \vee q)=\{C n(p \leftrightarrow q)\}$. And $H \perp(\phi \vee \psi) \subseteq H \perp[\phi, \psi]$ breaks down for non-theories $H$; just consider $H=\{p, q, p \vee q\}$, for which $H \perp(p \vee q)=\{\theta$ \} and $H \perp[p, q]=\{\{p \vee q\}\}$.
} 


$$
\begin{gathered}
\psi \in K-\langle S\rangle \text { iff } H^{\prime} \vdash \psi \text { for every } H^{\prime} \in H \Delta\langle S\rangle \neq \emptyset, \\
\text { or } H \Delta\langle S\rangle=\emptyset \text { and } \psi \in K . \\
\psi \in K-[S] \text { iff } H^{\prime} \vdash \psi \text { for every } H^{\prime} \in H \Delta[S] \neq \emptyset, \\
\text { or } H \Delta[S]=\emptyset \text { and } \psi \in K .
\end{gathered}
$$

\section{Prioritization}

At the end of Section 1.4 we expressed some dissatisfaction with the crudeness of the preference relation afforded there. This crudeness was due to our assumption that we only have syntactical information governing changes of belief. Now let us suppose that we do have more information. In every realistic situation, the elements of a belief base will differ in epistemic weight. Some of them are more important, more relevant, more plausible, more valuable, or more certain than others. Without fixing the precise interpretation, we can model this by a weak ordering (an asymmetric and modular ${ }^{6}$ relation) $\triangleleft$ of the elements of $H$. The strict relation $\triangleleft$ may be thought of as the asymmetric part of a transitive and connected relation $\unlhd$, or as being determined by an assignment of real numbers to the elements of $H$. The modularity of $\triangleleft$ essentially means that the relation $\bowtie$ of incomparability $(\phi \bowtie \psi$ iff neither $\phi \triangleleft \psi$ nor $\psi \triangleleft \phi$ ) is an equivalence relation and can thus be regarded as the relation of being tied or identical.

After Nebel [21], we understand by a level of priority (in Nebel's terms, a "degree of epistemic relevance") in a base $H$ an equivalence class in $H$ with respect to $\bowtie$. Levels of priority will be denoted $b y \phi$, where $\phi$ is an arbitrary representative of $\{\psi \in H$ : $\psi \bowtie \phi\}$. A level of priority $\phi$ is higher than a level of priority $\psi$, in symbols $\bar{\psi} \triangleleft \phi$, iff $\psi \triangleleft \phi$. It is clear that the ordering $\triangleleft$ between levels of priority is well-defined. The ordering $\triangleleft$ of $H$ generates a new ordering $\prec$ of the powerset of $H$, and indeed a new ordering of all sets of sentences:

DEFINITION 4. $H^{\prime} \prec H^{\prime \prime}$ iff there is a level of priority $\phi$ in $H$ such that $H^{\prime} \cap \phi \subset H^{\prime \prime} \cap \phi$ and for all higher levels of priority $\psi$ in $H$ we have $H^{\prime} \cap \psi \subseteq H^{\prime \prime} \cap \psi$

\footnotetext{
'A relation $\triangleleft$ is modular if $\phi \triangleleft \psi$ entails that either $\phi \triangleleft \chi$ or $\chi \triangleleft \psi$, for cvery $\chi$. Modularity and asymmetry entail transitivity.
} 
It is easy to check that $H^{\prime} \prec H^{\prime \prime}$ just in case there is a $\phi$ in $H^{\prime \prime}-H^{\prime}$ such that $\psi \triangleleft \phi$ for all $\psi$ in $H^{\prime}-H^{\prime \prime}$. Definition 4 , and in fact the entire method of prioritized base contraction, is very much in line with Rescher's "plausibility-tropic" Fundamental Rule of Presumption: "A positive presumption always favors the most plausible contentions among the available alternatives. It must stand until set aside by something yet more plausible..."7 [24, p.55]

From now on, $H \Delta \phi$ and $H \Delta[S]$ again denote the set of all maximal or "best" subsets of $H$ which fail to imply $\phi$, or respectively, any element of $[S]$, but maximality is now judged by the new preference relation $\prec$. These concepts generalize Alchourrón and Makinson's set $H \perp \phi$ which does not provide for differences in epistemic weight. If a belief base is not prioritized, i.e., if $\triangleleft$ is empty, then $\prec$ coincides with $\subset$ (relativized to $H$ ), and we get the so-called full meet base contraction as a limiting case. The basic idea of prioritized base contraction (Nebel) is to focus on $H \Delta \phi$ when looking for the rational way of discarding $\phi$. This is a reinterpretation of what we called meet contraction in Definition 2. Although it would in the case of (genuinely) prioritized belief bases be somewhat more suggestive to talk of "rational choices" or "best solutions" or "optimal selections" we shall continue to say that we follow the basic idea of minimal change.

The relation we have generated has the following properties:

OBSERVATION 1. The relation $\prec i s$

(i) a weak partial order (i.e., irreflexive and transitive),

(ii) base-specific in the sense that $H^{\prime} \prec H^{\prime \prime}$ iff $H^{\prime} \cap H \prec H^{\prime \prime}$ iff $H^{\prime} \prec H^{\prime \prime} \cap H$,

(iii) maximizing in the sense that $H^{\prime} \cap H \subset H^{\prime \prime} \cap H$ entails $H^{\prime} \prec H^{\prime \prime}$.

\footnotetext{
7 The procedure described in Rescher [23, pp. 50-51] is even more explicit. It is interesting, however, that Rescher in both [23, p. 46] and [24, pp. 15-16] requires his plausibility indexing to satisfy conditions similar to the ones used in the characterization of epistemic entrenchment. This is not at all necessary (neither is it detrimental, see Nebel [21, Theorem 9]). Also cf. Gärdenfors and Makinson [7, p. 88]: "The guiding idea for the construction is that when a knowledge system $K$ is revised or contracted, the sentences given up are those having the lowest degrees of epistemic entrenchment".
} 
If $\triangleleft$ is conversely well-founded (no infinite ascending chains), then $\prec$ is

(iv) stoppered in the sense that for every $H^{\prime} \subseteq H$ such that $C n(H) \cap S=\emptyset$ there is an $H^{\prime \prime} \in H \Delta[S]$ such that $H^{\prime \prime}=H^{\prime}$ or $H^{\prime}<H^{\prime \prime}$.

If $\triangleleft$ is not conversely well-founded, then $\prec$ may fail to be stoppered. even for singleton $S$.

Proof. Irreflexivity, transitivity, base-specificity and the maximizing property follow trivially from Definition 4.

Stopperedness: Assume that $C n\left(H^{\prime}\right) \cap S=\emptyset$ and that $\triangleleft$ is conversely well-founded. Since $\triangleleft$ is assumed to be modular, $\triangleleft$ over the levels of priority is a converse well-ordering, and thus conversely isomorphic to a unique ordinal $\tau$. Every level of priority $\psi$ can be associated with a unique ordinal $\sigma<\tau$, with smaller ordinals marking higher levels of priority. The level of priority with rank number $\sigma$ will be denoted $\psi_{\sigma}$. If $\sigma<\tau$ and $H_{\sigma}$ is a set such that $C n\left(H_{\sigma}\right) \cap S=0$, then let $H_{\sigma}^{*}$ be an arbitrary inclusion maximal subset of $\psi_{\sigma}$ such that $C n\left(H_{\sigma} \cup H_{\sigma}^{*}\right) \cap S=\emptyset$. By the compactness and monotonicity of $C n$, such $H_{\sigma}^{*}$ 's are easily shown to exist. They enter into our construction of $H^{\prime \prime}$ for stopperedness as follows:

$$
\begin{gathered}
H_{0}=H^{\prime} \\
H_{\sigma+1}=H_{\sigma} \cup H_{\sigma}^{*} \\
H_{\sigma}=\bigcup\left\{H_{\rho}: \rho<\sigma\right\} \text { for limit ordinals } \sigma .
\end{gathered}
$$

Finally, we put $H^{\prime \prime}=\bigcup\left\{H_{\sigma}: \sigma<\tau\right\}$. It is clear from the construction that either $H^{\prime} \prec H^{\prime \prime}$ or $H^{\prime \prime}=H^{\prime}$, and that $H^{\prime \prime}$ is in $H \Delta[S]$. And it follows from the compactness and monotonicity of $\mathrm{Cn}$ that for every $\sigma<\tau, C n\left(H_{\sigma}\right) \cap S=\emptyset$, and thus that $C n\left(H^{\prime \prime}\right) \cap S=\emptyset$. This completes the proof of the stopperedness of $\prec$.

If $\triangleleft$ is not required to be well-founded, then $\prec$ need not be stoppered, even in the case of a singleton $S$. We consider a propositional language with denumerably many atoms $\mathrm{p}, \mathrm{q}_{1}, \mathrm{q}_{2}$, $\mathrm{q}_{3}, \mathrm{q}_{4}, \ldots$ and the belief base $H=\left\{q_{\mathrm{i}} \vee q_{\mathrm{j}}: i, j=1,2,3, \ldots, i \neq j\right\} \cup$ $\left\{q_{\mathrm{i}} \rightarrow p: i=1,2,3, \ldots\right\}$ and ask for $H \Delta[\mathrm{p}]$. The prioritization is to be such that for all $i, j, k(j \neq k)$ we have $q_{\mathrm{i}} \rightarrow p \triangleleft q_{\mathrm{j}} \vee q_{\mathrm{k}}$ and for 
all $i<j$ we have $q_{\mathrm{i}} \rightarrow p \triangleleft q_{j} \rightarrow p$. Every $\prec$-maximal subset of $H$ which does not imply $p$ must contain $\left\{q_{i} \vee q_{j}: i \neq j\right\}$, because the disjunctions enjoy high priorities in $H$. And we are allowed to add exactly one material implication $q_{i} \rightarrow p$ without getting $p$. But since we are facing the infinite ascending chain $q_{1} \rightarrow p \triangleleft q_{2} \rightarrow p \triangleleft q_{3} \rightarrow p$

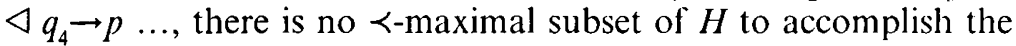
removal of $p$. Q.E.D. ${ }^{8}$

By the maximizing property, $H \Delta[S] \subseteq H \perp[S]$. The new preference relation $\prec$ admits finer distinctions than proper set inclusions. ${ }^{9}$ In the following we shall abstract from the special origin of the preference relation $\prec$ between subsets of $H$, that is, from the fact that $\prec$ was generated from $\triangleleft$ by the above construction. Instead we shall just draw on the properties of $\prec$ mentioned in $\mathrm{Ob}$ servation 1. In particular, we assume that $\prec$ is stoppered. Our level of generality thus lies somewhere between that of Nebel [20,21] and that of Hansson [12] who studies the still more abstract tool of selection functions. We shall always point out which of the properties we need for which of the things to prove.

Generalizing our notion of preference a bit, we say that a set $\mathcal{H}_{1}$ of sets of sentences is better than (or preferred to) a set $\mathcal{H}_{2}$ of sets of sentences, in symbols $\mathcal{H}_{2} \ll \mathcal{H}_{1}$, if for every $H_{2}$ in $\mathcal{H}_{2}$ there is an $H_{1}$ in $\mathcal{H}_{1}$ with $H_{2} \prec H_{1}$.

\footnotetext{
${ }^{8}$ This example disproves a claim of $\mathrm{Nebel}[21$, p. 56]. A similar counterexample has independently been provided by Weydert [30, p. 131].

${ }^{9}$ The importance of the maximizing property has been stressed repeatedly in the work of Sven Ove Hansson. - If base contractions are supplemented by a recovery-guarantecing appendage (sec Section 6.2 .2 below) and if everything is linite, prioritization is formally superfluous. It is shown in [28. Theorem 7] that then every prioritized base contraction can be simulated by a suitably chosen unprioritized belief base. But the simple bases to be used are grossly unintuitive. Consider for example the base $I=\{p, p \rightarrow q\}$ with the alternative prioritizations $p \rightarrow q \triangleleft p$ and $p \triangleleft p \rightarrow q$. In the former case, $H_{1}=\{p \wedge q, p, p \vee q, q \rightarrow p\}$, while in the latter case, $H_{2}=\{q, p \leftrightarrow q\}$, both without prioritization, will lead to precisely the same results as the prioritized belief base $I I$. In neither ease is there anything like a transparent connection with the original base $I I$.
} 


\section{Epistemic entrenchment}

The concept of epistemic entrenchment has turned out to be a natural and fruitful instrument for the analysis of belief change [7, $8,9,16,25,26,27]$. 'Epistemic entrenchment' is just another word for comparative retractability. Intuitively, $\phi<\psi$ means that it is easier to discard $\phi$ than to discard $\psi$. We may call this the basic idea of epistemic entrenchment. Below we shall offer two interpretations of this idea in order to make it more precise.

The relation between epistemic entrenchment and prioritization in a belief base has been first investigated by Bernhard Nebel. Initially he claimed that the latter is "fundamentally different" from the former [20, p. 308] but recently he changed his mind in favour of an interpretation of epistemic entrenchment as a special case of prioritization [21, pp. 53,68, 85]. We will join the former opinion in this paper. From the fact that $\phi$ has a higher priority than $\psi$ we may not infer that $\phi$ is better entrenched than $\psi$, nor may we rely on the reverse inference. ${ }^{10}$ This is true at least when an entrenchment relation is built up from a prioritized belief base in what we shall claim is the most natural fashion.

The most conspicuous difference, however, is that epistemic entrenchment relations are to respect the logical relationships between the beliefs in question, a feature that is completely absent in the concept of belief base priorities. The basic postulates an epistemic entrenchment relation < has to satisfy are:

(EE1) $T \nless T$

$\left(\mathrm{EE}^{\prime}\right)$ if $\phi<\psi$ and $\psi \vdash \chi$, then $\phi<\chi$

$\left(\mathrm{EE}^{\prime}\right)$ if $\phi<\psi$ and $\chi \vdash \phi$, then $\chi<\psi$

(EE3') if $\phi<\psi$ and $\phi<\chi$, then $\phi<\psi \wedge \chi$

$\left(\mathrm{EE} 3^{\downarrow}\right)$ if $\phi \wedge \psi<\psi$, then $\phi<\psi$
(Non-Triviality)

(Continuing Up)

(Continuing Down)

(Conjunction Up)

(Conjunction Down)

${ }^{10}$ See the counterexample against (EE6) in the proof of Observation 5 below. 
There is an equivalent and more economical set of postulates which does not refer to any connective of the object language. First, we can replace Non-Triviality by irreflexivity. Second, we note that postulates (EE2') and (EE3') taken together are equivalent to $\left(\mathrm{EE}^{\prime}\right)$, while $\left(\mathrm{EE}^{2}\right)$ and $\left(\mathrm{EE}^{\mathfrak{l}}\right)$ taken together are equivalent to $\left(\mathrm{EE}^{\mathfrak{l})}\right.$ [27]:

(EE') if $\phi<\psi$ for every $\psi$ in a non-empty set $S$ and $S \vdash \chi$, then $\phi<\chi$

(EE') if $\phi<\psi$ and $\{\psi, \chi\} \vdash \phi$ then $\chi<\psi$.

The set of basic postulates may be supplemented by the following ones.

(EE4) if $H$ is consistent, then: $\perp<\phi$ iff $H \vdash \phi$

(Minimality)

(EE5) if $H \phi$, then $\phi<T$

(Maximality)

(EE6) if $\phi<\psi$, then $\phi<\chi$ or $\chi<\psi$

(Modularity)

Again purely structural formulations of (EE4) and (EE5) are possible by substituting 'there is a $\psi$ such that $\psi<\phi$ (such that $\phi<\psi$ )' for ' $\perp<\phi$ ' (for ' $\phi<T$ '). For the motivation and discussion of all these postulates, see Gärdenfors and Makinson [9] and Rott [27]. Epistemic entrenchment relations are required to satisfy (EE1) $\left(\mathrm{EE}^{2}\right)$ in [27], and in addition (EE4) - (EE6) in [9]. (In fact, Gärdenfors and Makinson work with a non-strict relation $\leq$ which can be obtained from the strict relation < by taking the converse complement.)

Given a relation of epistemic entrenchment, how can we get a contraction function from it? For the principal case, where $\phi \in K$ and $\phi<T$, the standard definition $[9,27]$ is

DEFINITION 5. For any relation < of epistemic entrenchment, the large EE-contraction with respect to $<$ is given by

$$
\psi \in K \dot{-} \phi \text { iff } \psi \in K \text {, and } \phi<\phi \vee \psi \text { or } \vdash \phi
$$


The presence of the disjunction $\phi \vee \psi$ here is somewhat mysterious (to say the least). An alternative idea was ventilated in Rott [25]:

Definition 6. For any relation < of epistemic entrenchment, the small EE-contraction with respect to $<$ is given by

$$
\psi \in K \bullet \phi \text { iff } \psi \in K \text {, and } \phi<\psi \text { or } \vdash \phi
$$

Strictly speaking, both definitions ought to be supplemented by a clause stating that $K \dot{-} \phi=K$ if $\phi \notin K$ or $\phi \nless T$, but as this would not make a difference for the following, we omit it.

Both Definition 5 and Definition 6 make sure that $K \dot{-} \phi$ is a theory and that the contraction function - satisfies a number of rationality postulates. Large EE-contractions, but not small EE-contractions, satisfy the so-called postulate of recovery: $K \subseteq$ $(K \dot{-} \phi)+\phi$.

It follows from (EE2 $\left.{ }^{1}\right)$ that $K-\phi$ according to Definition 6 is a subset of $K \dot{-} \phi$ according to Definition 5-whence the names. Lindström and Rabinowicz [16, Section 5] argue convincingly to the effect that given an epistemic entrenchment relation $<$, any reasonable contraction of $K$ with respect to $\phi$ should result in a belief set which includes the small and is included in the large EEcontraction.

The basic idea of epistemic entrenchment is as yet still very vague and ought to be made more precise. The first or competitive interpretation of it suggests to determine the relative ease of retracting a sentence by looking at the fate of $\phi$ and $\psi$ in a direct competition between $\phi$ and $\psi$. It reconstructs epistemic entrenchment from observed contraction behaviour $[9,27]$ :

Difinition 7. For any contraction function - over $K$, the epistemic entrenchment relation revealed by - is given by

$$
\phi<\psi \text { iff } \psi \in K \dot{-}(\phi \wedge \psi) \text { and } \phi \notin K \dot{-}(\phi \wedge \psi) \text {. }
$$

Definition 7 yields extremely nice results when coupled with large 
EE-contraction functions over a theory $K$. If the contraction function - satisfies certain rationality postulates, then $<$ as obtained by Definition 7 is a relation of epistemic entrenchment from which we can recover - with the help of Definition 5. And conversely, if $<$ is a relation of epistemic entrenchment, then - as obtained by Definition 5 satisfies certain rationality postulates and permits a reconstruction of $<$ with the help of Definition 7. Details of that interplay can be found in Gärdenfors and Makinson [9] and Rott [27].

Another virtue of Definition 5 is the following. Nebel [21, proof of Theorem 9] showed that entrenchment relations satisfying (EE1) - (EE6) can be used as prioritizations of belief sets in the sense that they yield, via Definitions 2 and 4, the same result as when applied in Definition 5."

In [27], I emphatically adopt the idea that $K \dot{-}(\phi \wedge \psi)$ is to be interpreted as a multiple contraction, viz. the pick contraction with respect to $\{\phi, \psi\}$. Contracting $K$ with respect to $\phi \wedge \psi$, I argued, is exactly the same as retracting at least one of $\phi$ and $\psi$. In symbols, $K \dot{-}(\phi \wedge \psi)=K \dot{-}\langle\phi, \psi\rangle$. The motivation of Definition 7 is then clear. If you have to give up either $\phi$ or $\psi$, and you give up $\phi$ and keep $\psi$, then $\psi$ has been more entrenched than $\phi$.

I do not see any intuitive reason for supposing that the identity $K \dot{-}(\phi \wedge \psi)=K \dot{-}\langle\phi, \psi\rangle$ is inadequate in some applications. Still it is good to be prepared for this possibility. Another motive for modifying Definition 7 is that we want to avoid an explicit mentioning of particular connectives, in order to make the epistemic entrenchment approach applicable to restricted languages as encountered for instance in semantic networks. We take the motivation of Definition 7 seriously and suggest the following improvement:

11. An analogous applicability of entrenchment relations for so-called safe contractions is established in [26, Theorem 4(ii)]. Entrenchment relations are so wellbehaved that they appear to be appropriate for every contraction method without changing the results. 
DliFinITION 8. For any contraction function - over $K$, the epistemic entrenchment relation revealed by $-i$ s given by

$$
\phi<\psi \text { iff } \psi \in K-\langle\phi, \psi\rangle \text { and } \phi \notin K \dot{-}\langle\phi, \psi\rangle \text {. }
$$

This interpretation of the basic idea of epistemic entrenchment builds on the concept of pick contraction. Let us now try to deal with prioritized base contractions in terms of epistemic entrenchment.

\section{Prioritized base contractions as extended epistemic entrenchment contractions}

We extend the basic idea of epistemic entrenchment to sets of sentences. From now on, ' $\langle S\rangle \ll\langle T\rangle$ ' is intended to mean that it is easier to discard some element of $S$ than to discard some element of $T$. ' $[S] \ll[T]$ ' is intended to mean that it is easier to discard all elements of $S$ than to discard all elements of $T$. We shall speak of extended epistemic entrenchment in the sequel, with the two types pick and bunch entrenchment.

Let us try to widen the competitive interpretation of epistemic entrenchment accordingly. For pick entrenchment, this is easy. The obvious suggestion is

$$
\langle S\rangle \ll\langle T\rangle \text { iff } T \subseteq K \dot{\sim}\langle S \cup T\rangle \text { and } S \underline{\Xi} K \dot{-}\langle S \cup T\rangle \text {. }
$$

But for bunch entrenchment, there is no sensible condition which can be formalized with the present means. ${ }^{2}$

So we propose another understanding of-possibly extendedepistemic entrenchment. The second or minimal change interpretation of the basic idea of epistemic entrenchment builds on the basic idea of minimal change. It reads 'is easier' as 'does not require as great an informational loss as' or 'sacrifices less important beliefs than'. Formally, preference is judged by the relation ' $\prec$ ' of Section 2. Let us define the following version of extended epistemic entrenchment:

12. We do not wish to employ unheard-of objects such as $K \dot{ } \rightarrow\langle[S],[T]\rangle$. 
DEFINITION 9. For any base $H$ for $K$, the relation $\ll$ of bunch entrenchment generated by $H$ is defined by

$[S] \ll[T]$ iff for every $H^{\prime} \subseteq H$ such that $C n\left(H^{\prime}\right) \cap \mathrm{T}=0$ there is an $H^{\prime \prime} \subseteq H$ such that $H^{\prime} \prec H^{\prime \prime}$ and $C n\left(H^{\prime \prime}\right) \cap S=\emptyset$, and $H \psi$ for every $\psi$ in $S$.

The following equivalent formulation is sometimes more convenient:

OBSERVATION 2. Let $H$ be a base for $K$, and $\ll$ be the bunch entrenchment generated by $H$. Then $[S] \ll[T]$ iff for every $H^{\prime} \in H \Delta[T]$ there is an $H^{\prime \prime} \in H \Delta[S]$ such that $H^{\prime} \prec H^{\prime \prime}$, and $H \Delta[S] \neq \emptyset$, i.e., iff $H \Delta[T] \ll H \Delta[S] \neq \emptyset$.

Proof. From left to right. Take an $H^{\prime}$ from $H \Delta[\mathrm{T}]$. Since $C n(H) \cap \mathrm{T}=\emptyset$, the left hand side tells us that there is an $H^{\prime \prime}$ such that $C n\left(H^{\prime \prime}\right) \cap S=\emptyset$ and $H^{\prime} \prec H^{\prime \prime}$. By stopperedness, there is an $H^{*}$ in $H \Delta[\mathrm{S}]$ such that either $H^{*}=H^{\prime \prime}$ or $H^{\prime \prime} \prec H^{*}$. By transitivity, we have $H^{\prime} \prec H^{*}$, as desired. Now suppose that $H \Delta[\mathrm{S}]=\emptyset$. Then, by stopperedness, there is no $H^{\prime} \subseteq H$ such that $C n(H) \cap S=0$. Since we can take $H^{\prime}=\emptyset$, this entails that there is a $\psi$ in $S$ such that $\vdash \psi$.

From right to left. Take an $H^{\prime} \subseteq H$ such that $C n\left(H^{\prime}\right) \cap \mathrm{T}=\emptyset$. By stopperedness, there is an $H^{*}$ in $H \Delta[T]$ such that either $H^{*}=H^{\prime}$ or $H^{\prime} \prec H^{*}$. Now the right hand side tells us that there is an $H^{* *}$ in $H \Delta[S]$ such that $H^{*} \prec H^{* *}$. Clearly, $C n\left(H^{* *}\right) \cap S=\emptyset$, and by transitivity, $H^{\prime} \prec H^{* *}$, as desired. Now suppose that there is a $\psi$ in $S$ such that $\vdash \psi$. Then we have $H^{\prime} \vdash \psi$ for every $H^{\prime} \subseteq H$, so $H \Delta[S]$ is empty. Q.E.D.

We spare the reader an analogous definition of pick entrenchment, and we do not want to enter into a discussion of the properties of $\ll$. Our first result is that meet contractions of prioritized belief bases allow an elegant characterization in terms of bunch entrenchment.

ObSERVATION 3. Let $H$ be a base for $K,-$ be the meet contraction 
determined by $H$, and $\ll$ be the relation of bunch entrenchment generated by $H$. Then $\psi \in K-\phi$ iff $[\phi] \ll[\phi, \psi]$.

Proof. The trick is to reformulate the principal case of Definition 2 , that is, the case where $H \phi$, in the following way:

$$
\psi \in K \dot{-} \phi
$$

for every $H^{\prime} \subseteq H$ such that $H^{\prime} H \phi$ and there is no

$H^{\prime \prime} \subseteq H$ such that $H^{\prime} \prec H^{\prime \prime}$ and $H^{\prime \prime} H \phi$, it holds that $H^{\prime} \vdash \psi$

for every $H^{\prime} \subseteq H$ such that $H^{\prime} H \phi$ and $H^{\prime} H \psi$ there is an $H^{\prime \prime} \subseteq H$ such that $H^{\prime} \prec H^{\prime \prime}$ and $H^{\prime \prime} H \phi$

for every $H^{\prime} \in H \Delta[\phi, \psi]$ there is an $H^{\prime \prime} \in H \Delta[\phi]$ such that $H^{\prime} \prec H^{\prime \prime}$

$$
H \Delta[\phi, \psi] \ll H \Delta[\phi]
$$

$$
[\phi] \ll[\phi, \psi]
$$

We can directly represent meet contractions of belief bases as extended EE-contractions in the following sense.

DEFINITION 10. For any relation $\ll$ of bunch entrenchment, the EEE-contraction with respect to $\ll$ is given by

$$
\psi \in K \dot{-} \phi \text { iff } \psi \in K \text {, and }[\phi] \ll[\phi, \psi] \text { or } \vdash \phi
$$

Like Definition 6, this definition is connective-free. It relates two fundamentally different ideas. If $\ll$ is determined by Definition 9 ,

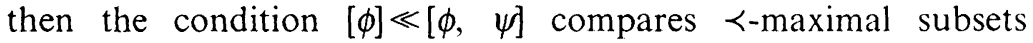
of $H$ which do not imply $\phi$ with $\prec$-maximal subsets of $H$ which imply neither $\phi$ nor $\psi$. In a sense, the definition involves maxichoice bunch contraction: $\psi$ is in $K-\phi$ iff for every $\gamma$ there is a $\gamma^{\prime}$ such that the maxichoice contraction of $K$ with respect to $\phi$ 
determined by $H$ and $\gamma^{\prime}$ is better than the maxichoice contraction of $K$ with respect to $\phi$ and $\psi$ determined by $H$ and $\gamma$. On the other hand, Definition 10 characterizes meet contraction by single sentences, as shown in Observation 3.

\section{Epistemic entrenchment generated by prioritized belief bases}

Given a prioritized base $H$ for a belief set $K$, we now try to find a more familiar, i.e., non-extended, relation of epistemic entrenchment, without beforehand committing ourselves to a certain contraction method.

\subsection{Positive relations of "epistemic entrenchment"}

Let us for the sake of simplicity assume in this section that all members of $H$ are of equal epistemic value, i.e., that there is no prioritization (empty $\triangleleft$ ). A first idea of extracting an epistemic entrenchment relation from a base $H$ for a belief set $K$ is the following. Call a set $H^{\prime}$ a proof set for $\phi$ iff $H^{\prime} \vdash \phi$. Then $\psi$ might be called more entrenched than $\phi$ (in $K=C n(H)$ ) if and only if for every proof set $H^{\prime} \subseteq H$ for $\phi$ there is a proof set $H^{\prime \prime} \subseteq H$ for $\psi$ which is "better than" $H^{\prime}$. This is a positive interpretation of epistemic entrenchment, focussing on the ways to gain a belief. A variant of this idea is successful in the reconstruction of "safe contractions" of belief sets with the help of EE-contractions [2, 26]. But what does 'better than' mean, if we have no prioritization? According to the line of thought developed above, a proof set is better than another if it contains fewer explicit beliefs. That is to say that $H^{\prime \prime}$ is better than $H^{\prime}$ if $H^{\prime \prime} \subset H^{\prime}$. So our first idea amounts to putting

$$
\begin{gathered}
\phi<\psi \text { iff for every } H^{\prime} \subseteq H \text { such that } H^{\prime} \vdash \phi \text { there is an } I^{\prime \prime} \\
\text { such that } H^{\prime \prime} \subset H^{\prime} \text { and } H^{\prime \prime} \vdash \psi
\end{gathered}
$$

However, we shall see that this is not the right approach to the present problem. The relation defined satisfies (EE1), (EE2') and $\left(E E 2^{l}\right)$, but neither $\left(E E 3^{\prime}\right)$ nor $\left(E^{2} 3^{\downarrow}\right)$. The same is true for other "positive" suggestions such as the weaker 
$\phi<\psi$ iff for every $H^{\prime} \subseteq H$, if $H^{\prime} \vdash \phi$, then $H^{\prime} \vdash \psi$, and there is an $H^{\prime \prime} \subseteq H$ such that $H^{\prime \prime} \vdash \psi$, but $H^{\prime \prime} H \phi$.

The main fault of these positive relations is that they do not conform to the basic idea of epistemic entrenchment. In order to judge the relative entrenchments of $\phi$ and $\psi$ in meet contractions of belief bases, we have to look at the subsets of $H$ which do not entail $\phi$ or $\psi$, rather than at the proof sets for $\phi$ or $\psi$.

\subsection{Epistemic entrenchment from belief bases: a negative interpretation}

We return to the basic idea of epistemic entrenchment. As it happens, the competitive and the minimal change interpretation of it can be unified in the present case. For our official definition, we employ the latter one:

Definition 11. For any prioritized base $H$ for $K$, the relation of epistemic entrenchment generated by $H$ is given by

$\phi<\psi$ iff $H \phi$ and for every $H^{\prime} \subseteq H$ such that $H^{\prime} H \psi$ there is an $H^{\prime \prime} \subseteq H$ such that $H^{\prime} \prec H^{\prime \prime}$ and $H^{\prime \prime} H \phi$.

This is a singleton version of Definition 9. Clearly, it is a negative interpretation of epistemic entrenchment, focussing on the ways to discard a belief. It is intuitively well-motivated. Roughly, $\psi$ is more entrenched than $\phi$ if and only if for every way of discarding $\psi$ there is a better way of discarding $\phi$.

As a special case of Observation 2, we take down

OBSFRVATION 4. Let $H$ be a prioritized base for $K$, and $<$ be generated by $H$. Then $\phi<\psi$ iff $H \phi$ and for every $H^{\prime} \in H \Delta \psi$ there is an $H^{\prime \prime} \in H \Delta \phi$ such that $H^{\prime} \prec H^{\prime \prime}$, i.e., iff $H \Delta \psi \prec H \Delta \phi \neq \emptyset$.

So $\psi$ is more entrenched than $\phi$ if for every optimal way of discarding $\psi$ there is a still better optimal way of discarding $\phi$. More exactly, in terms of maxichoice contraction functions, if for every $\gamma$ there is a $\gamma^{\prime}$ such that the maxichoice contraction of $K$ with 
respect to $\phi$ determined by $H$ and $\gamma^{\prime}$ is preferable to the maxichoice contraction of $K$ with respect to $\psi$ determined by $H$ and $\gamma$.

In the following, we trace some of the implications of Definition 11. In 5.3, we verify that Definition 11 generates a relation of epistemic entrenchment in the generalized sense of Rott [27], but not in the standard sense of Gärdenfors and Makinson [9]. Next, in 5.4, we show that for meet contractions the two interpretations of the basic idea of epistemic entrenchment coincide. In 5.5, we compare Definition 11 with entrenchment relations obtained by combining suggestions from Rott [25] with the special preference relation $\prec$ of Section 2. In Section 6.1, we show that meet contractions, which are EEE-contractions characterized by Definitions 9 and 10 , can be interpolated by means of small and large EE-contractions based on the epistemic entrenchment relation generated by the base. Although the approximation cannot in general be strengthened to an identity, a perfect agreement can be attained in a number of important special cases, as is shown in Section 6.2.

\subsection{A prioritized belief base generates a generalized, but not a standard relation of epistemic entrenchment}

It is easy to verify that the relation $<$ defined in Definition 11 has the following properties:

ObSERVATION 5. For every prioritized belief base $H$, the relation < generated by $H$ satisfies (EEl) - (EE3 $\left.3^{\mathfrak{l}}\right)$ and $(E E 4)-(E E 5)$, but it does not satisfy (EE6).

That is, Definition 11 does not yield an epistemic entrenchment relation in the sense of Gärdenfors and Makinson [9], but it does yield an epistemic entrenchment relation in the less demanding sense of Rott [27].

Proof. (EE1) through (EE3') are obvious from Definition 11. For (EE3 $\left.3^{\lfloor}\right)$, assume that for every $H^{\prime}$ with $H^{\prime} H \psi$ there is an $H^{\prime \prime}$ with $H^{\prime} \prec H^{\prime \prime}$ and $H^{\prime \prime} H \phi \wedge \psi$. We have to show that for every such $H^{\prime}$ there is an $H^{*}$ with $H^{\prime} \prec H^{*}$ and $H^{*} H \phi$. Now for every such $H^{\prime}$, we have $H^{\prime} H \phi \wedge \psi$. By stopperedness, there is an $H^{*}$ in $H \Delta(\phi \wedge \psi)$ 
such that either $H^{*}=H^{\prime}$ or $H^{\prime} \prec H^{*}$. By our assumption and the maximality of $H^{*}, H^{*} \vdash \psi$. Since $H^{*} H \phi \wedge \psi$, it follows that $H^{*} H \phi$. For (EE4), observe that for every consistent $H$, we have $H \Delta \perp=$ $\{H\}$, by the maximizing property. So $\perp<\phi$ means that $H$ is preferred to every $H^{\prime}$ in $H \Delta \phi$. For (EE5), observe that $\phi<T$ is equivalent to $H \phi$.

Counterexample to (EE6): The belief base $H=\{p, p \vee q, \neg p \vee q\}$ with no prioritization (empty $\triangleleft$ ) has $H \Delta p=\{\{p \vee q, \neg p \vee q\}\}, H \Delta q$ $=\{\{p, p \vee q\},\{\neg p \vee q\}\}$, and $H \Delta(p \vee q)=\{\{\neg p \vee q\}\}$. This gives us $p<p \vee q$, but neither $p<q$ nor $q<p \vee q$. Q.E.D.

The relation < generated in the counterexample to (EE6) is further discussed as Example 1 in Rott [27]. It is worth noting that here we have $p<p \vee q$ although not $p \triangleleft p \vee q$. Moreover, if we add $q$ to the base $H$, then the generated entrenchment relation < still fails to verify $q<p$-although we may well have $q \triangleleft p$. We realize once again that prioritization and entrenchment are indeed two altogether different kinds of thing.

\subsection{The Coincidence Lemma}

If pick contractions are formalized as in Definition 3, Definition 11 turns out to be tantamount to the first interpretation of the basic idea of epistemic entrenchment as formalized in Definition 8. In the context of prioritized base contraction, the competitive and the minimal change interpretations of epistemic entrenchment coincide.

Observation 6. (CoIncidence Lemma) Let $H$ be a prioritized base for $K$, let $<$ be the epistemic entrenchment relation generated by $H$ and $<^{\prime}$ be the epistemic entrenchment relation revealed by the pick version of multiple meet contraction. Then $\phi<\psi$ iff $\phi<^{\prime} \psi$.

Proof.

$$
\phi<^{\prime} \psi
$$

iff (by Def. 8)

$$
\psi \in K \dot{-}\langle\phi, \psi\rangle \text { and } \phi \notin K \dot{-}\langle\phi, \psi\rangle
$$

$H^{\prime} \vdash \psi$ for every $H^{\prime} \in H \Delta\langle\phi, \psi\rangle$, and $H^{\prime} \nvdash \phi$

for some $H^{\prime} \in H \Delta\langle\phi, \psi\rangle$ 
$H^{\prime} \notin H \Delta\langle\phi, \psi\rangle$ for every $H^{\prime}$ such that $H^{\prime} H \psi$, and

$$
H \phi \wedge \psi
$$

iff

$H^{\prime} \notin H \Delta\langle\phi, \psi\rangle$ for every $H^{\prime} \in H \Delta \psi$, and $H \phi \wedge \psi$

iff

for every $H^{\prime} \in H \Delta \psi$ there is an $H^{\prime \prime} \subseteq H$ with $H^{\prime} \prec H^{\prime \prime}$ and $H^{\prime \prime} H \phi$, and $H \phi \wedge \psi$

iff

for every $H^{\prime} \in H \Delta \psi$ there is an $H^{\prime \prime} \in H \Delta \phi$ such that

$$
H^{\prime} \prec H^{\prime \prime} \text {, and } H \phi
$$

iff

$H \Delta \psi \prec H \Delta \phi$ and $H \phi$

iff (by Def. 11)

$$
\phi<\psi
$$

Q.E.D.

Intuitively this result is just what one may expect. It is still interesting, however, because it shows the confluence of two formally quite different ideas. As shown in Observation 4, Definition 1.1 refers to $\prec$-maximal non-implying subsets, i.e., to maxichoice contractions with respect to $\phi$ and $\psi$, but multiple contractions are not involved. On the other hand, Definitions 3 and 8 analyze epistemic entrenchment in terms of meet pick contractions (which could, however, also be presented as meet singleton contractions with respect to $\phi \wedge \psi)$.

\subsection{More ways to generate the relation of epistemic entrenchment}

There are still more ways to arrive at the determination of epistemic entrenchment given by Definition 11. We follow a line of reasoning that has been proposed in the context of theory contraction.

5.5.1. First let us combine Rott's [25] way of constructing epistemic entrenchment from a preference relation over maximal non-implying subsets of theories with an understanding of 'better' as 'preserving more important beliefs'.

According to Definition 5 of [25], $\phi \leq \psi$ if and only if for all 
$K^{\prime} \in K \perp \psi$ there is a $K^{\prime \prime} \in K \perp \phi$ which is at least as good as $K^{\prime}$ (remember that $K \perp \phi$ is the set of all inclusion maximal subsets of $K$ that do not imply $\phi$ ). For the transition from $\leq$ to $<$ we take the converse complement. So $\phi<\psi$ if and only if there is a $K^{\prime \prime} \in K \perp \phi$ such that all $K^{\prime \prime} \in K \perp \psi$ are worse than $K^{\prime} \cdot{ }^{13}$ But this is not yet satisfactory. Because we must allow for the possibility of elements in $M(K)=\bigcup\{K \perp \chi: \chi \in K-C n(\emptyset)\}$ which are incomparable with respect to the preference relation under consideration, this condition seems too demanding. We should rearrange the quantifier phrases from $\exists \forall$ to $\forall \exists .{ }^{14}$ Our official proposal then is this:

$$
\begin{gathered}
\phi<\psi \text { iff } \forall \phi \text { and for all } K^{\prime} \in K \perp \psi \text { there is a } \\
K^{\prime \prime} \in K \perp \phi \text { which is better than } K^{\prime} \text {. }
\end{gathered}
$$

Now we recall the basic idea of prioritized base contraction: For sets $K^{\prime}$ and $K^{\prime \prime}$ of sentences (in $M(K)$ ), $K^{\prime \prime}$ is better than $K^{\prime}$, in symbols $K^{\prime} \prec K^{\prime \prime}$, iff there is a level of priority in the base $H$ such that at this level $K^{\prime \prime}$ exceeds $K^{\prime}$, while at every higher level $K^{\prime \prime}$ is at least as comprehensive as $K^{\prime}$.

It should be noted that $\prec$ so defined is not a preference relation of the kind discussed in [25], because it is not modular, i.e., its converse complement does not satisfy transitivity. Putting the last two definitions together, we have a new characterization of epistemic entrenchment which turns out to be equivalent to Definition 11 .

OBSERVATION 7. Let $H$ be a prioritized base for $K$. Then the following two conditions are equivalent:

(i) for every $K^{\prime} \in K \perp \psi$ there is a $K^{\prime \prime} \in K \perp \phi$ such that $K^{\prime} \prec K^{\prime \prime}$,

(ii) for every $H^{\prime} \in H \Delta \psi$ there is an $H^{\prime \prime} \in H \Delta \phi$ such that $H^{\prime} \prec H^{\prime \prime}$.

\footnotetext{
${ }^{13}$ I am cheating a little at this point. I substitute 'worse than' for 'not at least as good as'-a move which would obviously require connectivity for the relation 'at least as good as'.

${ }^{14}$ For reasons which cannot be explained here, I now believe that a reversal of the quantilier phrases in Rott [25] is more adequate anyway.
} 
Proof. (i) implies (ii): Let $H^{\prime} \in H \Delta \psi \subseteq H \perp \psi$. Extend $H^{\prime}$ to a set $K^{\prime} \in K \perp \psi$. This is possible and gives us $K^{\prime} \cap H=H^{\prime}$. Take a $K^{\prime \prime}$ from (i) and put $H^{\prime \prime \prime}=K^{\prime \prime} \cap H$. Since $K^{\prime} \prec K^{\prime \prime}$, we get $H^{\prime} \prec H^{\prime \prime \prime}$, by base-specificity. Furthermore, we have $H^{\prime \prime} \subseteq H$ and $H^{\prime \prime \prime} H \phi$. So by stopperedness, there is an $H^{\prime \prime}$ in $H \Delta \phi$ such that $H^{\prime \prime} \prec H^{\prime \prime}$ or $H^{\prime \prime \prime}=H^{\prime \prime}$. By transitivity, $H^{\prime} \prec H^{\prime \prime}$, as desired.

(ii) implies (i): Let $K^{\prime} \in K \perp \psi$, and put $H^{\prime \prime \prime}=K^{\prime} \cap H$. We have $H^{\prime \prime \prime}$ $\subseteq H$ and $H^{\prime \prime \prime} H \psi$. So by stopperedness, there is an $H^{\prime}$ in $H \Delta \psi$ such that $H^{\prime \prime \prime} \prec H^{\prime}$ or $H^{\prime \prime \prime}=H^{\prime}$. Take an $H^{\prime \prime}$ from (ii). By transitivity, $H^{\prime \prime} \prec H^{\prime \prime}$. Extend $H^{\prime \prime} \in H \Delta \phi \subseteq H \perp \phi$ to a set $K^{\prime \prime} \in K \perp \phi$. This is possible and gives us $K^{\prime \prime} \cap H=H^{\prime \prime}$. Since $K^{\prime} \cap H=H^{\prime \prime} \prec H^{\prime \prime}=K^{\prime \prime} \cap H$, we get, by base-specificity, $K^{\prime} \prec K^{\prime \prime}$, as desired. Q.E.D.

5.5.2. There is also a method to derive epistemic entrenchment from a preference relation over arbitrary sets of sentences. According to [25, Lemma 2(iii)], $\phi \leq \psi$ if and only if for all sets $S$ such that $S H \psi$ there is a $\mathrm{T}$ such that $\mathrm{T} H \phi$ and $\mathrm{T}$ is at least as good as $S$. Now let us take the converse of complement and adjust the quantifier arrangement in a way similar to the previous section. What we get is

$$
\begin{gathered}
\phi<\psi \text { iff } H \phi \text { and for all sets } S \text { such that } S H \psi \text { there is a } T \\
\text { such that } T H \phi \text { and } T \text { is better than } S \text {. }
\end{gathered}
$$

Combining this with an understandig of 'better' as ' $>$ ', we discover yet another equivalent form of Definition 11:

ObSeRvation 8. Let $H$ be a prioritized base for $K$. Then the following two conditions are equivalent:

(i) for every $S$ such that $S H \psi$ there is a $T$ such that $T H \phi$ and $S \prec T$,

(ii) for every $H^{\prime} \subseteq H$ such that $H^{\prime} H \psi$ there is an $H^{\prime \prime} \subseteq H$ such that $H^{\prime \prime} H \phi$ and $H^{\prime} \prec H^{\prime \prime}$.

Proof. (i) implies (ii): Take $H^{\prime \prime}=T \cap H$ and apply base-specificity. (ii) implies (i): Put $H^{\prime}=S \cap H$, take $T=H^{\prime \prime}$ and apply base-specificity. Q.E.D. 


\section{Prioritized base contractions as approximated by epistemic entrenchment contractions}

Suppose that the epistemic entrenchment relation $<$ is generated by the prioritized belief base $H$ for $K$. We wonder about the relation between the meet contractions generated by $H$ on the one hand and the large and small EE-contractions based on $<$ on the other. Is it possible to get what we got by extended epistemic entrenchment above (Observation 3) with the help of singleton epistemic entrenchment? As for large EE-contractions, the answer must be negative, because they are known to satisfy the recovery postulate, which base contractions notoriously do not.

\subsection{The Interpolation Lemma}

It turns out that singleton epistemic entrenchment is insufficient in general, but an approximation by upper and lower bounds is possible. The entrenchment relation determined by a belief base $H$ with the help of Definition 11 allows us to follow the above-mentioned recommendation of Lindström and Rabinowicz. We can interpolate meet changes according to Definition 2, i.e., EEEchanges according to Definitions 9 and 10 , by means of large and small EE-changes according to Definitions 5 and 6.

OBSHRVATION 9 (InTERPOLATION LEMma). Let $H$ be a prioritized base for $K$ and $<$ be the entrenchment relation generated by $H$. Furthermore, let - be the meet contraction function determined by $\mathrm{H}$, let - , be the small and $-_{2}$, be the large EE-contraction with respect to $<$. Then

$$
K{ }_{1} \phi \subseteq K \dot{-} \phi \subseteq K \dot{ }_{2} \phi
$$

The converse inclusions are not valid, even if there is no prioritization.

Proof. Let $H, K$ and $<$ be as indicated. If $\vdash \phi$, there is nothing to prove because then $K \dot{-} \phi=K$ by definition in every case. So let $H \phi$ and $\psi \in K$. Unravelling the definitions, we find that 
MODELLINGS FOR BELIEF CHANGE:

$$
\begin{array}{ccc}
\psi \in K{ }_{1} \phi & \text { iff } & H \Delta \psi \ll H \Delta \phi \\
\psi \in K-\phi & \text { iff } & H \Delta[\phi, \psi] \ll H \Delta[\phi] \\
\psi \in K{ }_{2} \phi & \text { iff } & H \Delta(\phi \vee \psi) \ll H \Delta \phi
\end{array}
$$

But now we see that our claim is obvious because by

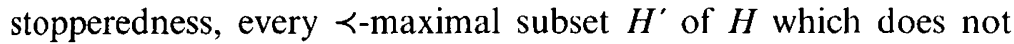
imply $\phi \vee \psi$ is identical with or $\prec$-dominated by some $\prec$-maximal subset $H^{\prime \prime}$ of $H$ which implies neither $\phi$ nor $\psi$, and every such $H^{\prime \prime}$ is in turn identical with or $\prec$-dominated by some $\prec$-maximal subset $H^{\prime \prime \prime}$ of $H$ which does not imply $\psi$.

The converse inclusions are not valid, even if we assume that there is no prioritization: If $H=\{p, q\}$ and $\triangleleft$ is empty, then $q \in K \dot{-} p$ according to (II), but not according to (I). If $H=\{p \wedge q$, $p \vee q\}$ and $\triangleleft$ is empty, then $q \in K \dot{-} p$ according to (III), but not according to (II). (Notice that if $H=\{p, p \vee q\}$, the absurd result $q \in K \dot{-} p$ according to (III) is avoided by the requirement that $q$ be contained already in $K=C n(H)$.) Intuitively, the meet contraction according to (II) gives the desired results. Q.E.D.

\subsection{Perfect agreements}

In a couple of important cases, the correspondence between meet contractions and epistemic entrenchment contractions is perfect, if the latter are to mean large EE-contractions based on the relation $<$ generated by the prioritized belief base $H$. There are at least three ways to obtain an equivalence: when a prioritized belief base is logically closed, when a contraction function is blown up so as to guarantee the recovery property, and when a contraction is used only as an intermediate step in bringing about a belief revision.

6.2.1. Closed bases. The first case is when the base $H$ is already a belief set, i.e., when $H=K$. Then (II) and (III) turn out to be equivalent for every $\psi$ in $K$. We already know that (II) implies (III). Suppose for reductio that (III) is true and (II) is false. From the latter we know by stopperedness and transitivity that there is a $K^{\prime}$ in $K \Delta[\phi, \psi]$ for which there is no better set not implying $\phi$. Thus $K^{\prime}$ is in $K \Delta \phi$. By (III), $K^{\prime}$ cannot be in $K \Delta(\phi \vee \psi)$. So since every

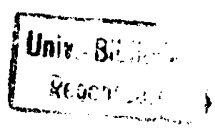


set preferred to $K^{\prime}$ implies $\phi \vee \psi, K^{\prime} \vdash \phi \vee \psi$. But also $\phi \rightarrow \psi \in K^{\prime}$, by $K^{\prime} \in K \Delta \phi \subseteq K \perp \phi$ and $\psi \in K-K^{\prime} .^{15}$ Hence $K^{\prime} \vdash \psi$, contradicting $K^{\prime} \in K \Delta[\phi, \psi]$.

If there is no prioritization, i.e., if $\triangleleft$ is empty and $\prec$ is $\subset$, this case is of limited relevance. Then the epistemic entrenchment relation generated by $K$ is nearly empty if $K$ is a theory, because then $K^{\prime} \in K \Delta \psi=K \perp \psi$ and $K^{\prime \prime} \in K \Delta \phi=K \perp \phi$ imply $K^{\prime} \not \subset K^{\prime \prime} .{ }^{16}$ So in this case, $\phi<\psi$ according to Definition 11 can hold only if either $\psi \in K$ and $\phi \notin K$, or $\vdash \psi$ and $H \phi$. Similarly, for theories $K$ without prioritization, (I) is only possible if either $\psi \in K$ and $\phi \wedge \psi \notin K$, or $\vdash \psi$, and (III) is only possible if either $\phi \vee \psi \in K$ and $\phi \notin K$, or $\vdash \phi \vee \psi$. This corresponds to a well-known trivialization result of Alchourrón and Makinson [1, Observation 2.1] for full meet contractions of theories. However, if a theory is genuinely prioritized, the construction offered is perfectly reasonable.

6.2.2. Nebel's blown-up contractions. The second case in point is when a meet contraction is supplemented with a mechanism to enforce the recovery postulate. This is basically the suggestion of Nebel [20]:

Definition 12. For any prioritized base $H$ for $K$, the blown-up meet contraction - is given by

$$
\begin{gathered}
\psi \in K \dot{-\phi} \text { iff }(K \dot{-}, \phi) \cup\{\phi \rightarrow \chi: \chi \in H\} \vdash \psi \text {, where } K \dot{-}, \phi \\
\text { is the meet contraction determined by } H \text {. }
\end{gathered}
$$

The set $\operatorname{Rec}=\{\phi \rightarrow \chi: \chi \in H\}$ is a recovery ticket which allows one to "undo" a base contraction with respect to $\phi$. It is easy to check that on Definition $12, K=(K-\phi)+\phi$, for every $\phi$ in $K$. But since clearly $\neg \phi$ implies every element of $R e c$, and $R e c$ in turn implies $\phi \rightarrow \psi$ (for every $\psi$ in $K$ ), we find that $\psi \in K-\phi$ according to Definition 12 iff $\phi \vee \psi \in K \dot{-} \phi$ according to Definition 2. Comparing (II) with (III) and realizing that $H \Delta[\phi, \phi \vee \psi]=H \Delta(\phi \vee \psi)$, we see that this is the same as $\psi \in K \dot{-} \phi$ according to Definitions 5

\footnotetext{
15 See footnote 5 .

${ }^{16}$ Unless $K$ " equals $K$. Cf. footnote 5 .
} 
and 11. So Nebel's variation on meet contractions is captured exactly by large EE-contractions based on the relation $<$ generated by $H$.

6.2.3. Revisions based on the Levi identity. Thirdly, the correspondence is perfect if a contraction is only an intermediate for a revision constructed with the help of the Levi identity. Since, by Levi and the deduction theorem, $\psi$ is in $K * \phi$ iff $\phi \rightarrow \psi$ is in $K \sqcup \neg \phi$, we have to check $K-\neg \phi$ only for sentences of the form $\phi \rightarrow \psi$. But clearly, for every relation $<$ satisfying (EE2'), $\neg \phi<(\phi \rightarrow \psi)$ is equivalent to $\neg \phi<\neg \phi \vee(\phi \rightarrow \psi)$, so Definitions 5 and 6 are equivalent for sentences of the form $\phi \rightarrow \psi$ in $K \sqcup \neg \phi$. Hence, by the Interpolation Lemma, both large and small EE-revisions are identical with the meet revision determined by $H .^{17}$

\section{Conclusion}

The aim of this paper has not been to present exciting new technical results. It is rather meant to provide one more illustration for the versatility of the concept of epistemic entrenchment in the rational reconstruction of belief change. To the best of our knowledge, we have offered one of the first suggestions how to apply epistemic entrenchment to belief states ("bases") which are not supposed to be logically closed (compare Dubois and Prade [4]) but which may be partitioned into various levels of priority. We also hope to have furthered the intuitive understanding of epistemic entrenchment and its relation to multiple contraction.

Our starting point has been a fixed prioritized belief base $H$. It contains the reasoner's explicit beliefs of various strengths and generates a belief set $K=C n(H)$. Our concern is "syntax-based" belief change, or belief change determined by belief bases, and we assume that it is the syntactical structure and the epistemic weighting of the explicit beliefs that governs the changes of $K$.

We have given a reformulation of meet contractions determined by a prioritized belief base as extended EE-contractions: Definition 2 is equivalent to the combination of Definitions 9 and 10 .

${ }^{17}$ Presumably because of the problems with the recovery postulate, Nebel's attention switches from contractions in [20] to revisions in [21]. 
This representation depends on an extension of the concept of epistemic entrenchment to sets of sentences ("bunch entrenchment"). We elaborated on the basic idea of epistemic entrenchment as comparative retractability by giving it two different readings. The usual "competitive" interpretation was distinguished from what we called the "minimal change interpretation" of the phrase ' $\psi$ is harder to discard than $\phi$ '.

We proposed a method of extracting an epistemic entrenchment relation < from a prioritized belief base $H$. Discovering that Definition 11 is equivalent to the combination of Definitions 3 and 8 , we observed a confluence of the two interpretations of epistemic entrenchment (the "Coincidence Lemma"). It was demonstrated that for every meet contraction determined by a prioritized base $H$ one can specify upper and lower bounds in the form of large and small EE-contractions based on the relation < generated by $H$ (the "Interpolation Lemma"). In a number of interesting cases, this result can be sharpened to an identity. Nebel [21, Theorem 9] proved that an epistemic entrenchment relation < can be applied as if it were a belief base prioritization $\triangleleft$. We have offered an answer to the reverse question: Given some belief base prioritized by $\triangleleft$, is it possible to exhibit a natural relation of entrenchment $<$ such that the EE-contraction with respect to $<$ leads to the same results as the meet contraction with respect to $\triangleleft$ ?

Since the publication of Gärdenfors's Knowledge in Flux, relations of epistemic entrenchment have been known to be interdefinable with belief contractions. For theory change by singletons, the following transitions are standard in the literature:

$$
\begin{gathered}
\phi<\psi \text { iff } \psi \in K \dot{-}(\phi \wedge \psi) \text { and } \phi \notin K \dot{-}(\phi \wedge \psi) \\
\psi \in K \dot{-} \text { iff } \psi \in K \text {, and } \phi<\phi \vee \psi \text { or } \vdash \phi
\end{gathered}
$$

To my mind, there is no denying that these bridge principles are the pivotal points of an illuminating and well-developed theory of belief change [7, 9, 25, 26, 27]. In our particular framework where everything starts from a prioritized belief base, one can prove that the principles hold, but only approximately in some cases. In order to bring this out as clear as possible, we adopt the following 
notation. For any prioritized belief base $H$, let $C(H)$ and $\mathcal{E}(H)$ be the contraction operation and the entrenchment relation derived from $H$ by Definitions 2 and 11 respectively. Moreover, if $<$ is an entrenchment relation and - is a contraction function, let $C^{\prime}(<)$ and $\mathcal{E}(\dot{-})$ denote the contraction function and the entrenchment relation obtained by the above bridge principles (which are identical with our Definitions 5 and 7). Finally, let $C(<)$ denote the small EE-contraction with respect to $<$.

We are now in a position to formulate the following summary of relationships:

OBSERVATION 10. Let $H$ be a prioritized belief set and let $-=C(H)$ and $<=\mathcal{E}(H)$. Then

(a) $\mathcal{E}(\dot{-})=<$,

(b) $C^{-}(<) \subseteq-\subseteq C^{+}(<),{ }^{18}$

(c) $C^{-}(\mathcal{E}(\dot{-})) \subseteq-\subseteq C^{+}(\mathcal{E}(\dot{-}))$,

(d) $\mathcal{E}\left(C^{-}(<)\right)=<=\mathcal{E}\left(C^{+}(<)\right)$.

Proof: (a) is the Coincidence Lemma, and (b) is the Interpolation Lemma; (c) follows from (a) and (b): $C^{-}(\mathcal{E}(\dot{-}))=C^{-}(\mathcal{E}(C(H)))=$ (by (a)) $C^{-}(\mathcal{E}(H)) \subseteq$ (by (b)) $C(H) \subseteq$ (by (b) $C^{+}(\mathcal{E}(H)) \subseteq$ (by (a)) $C^{+}(\mathcal{E}(C(H)))=C^{+}(\mathcal{E}(-))$; for $(\mathrm{d})$, let $<^{\prime}=\mathcal{E}(C(<))$ and $<^{\prime \prime}=$ $\mathcal{E}\left(C^{+}(<)\right)$. Then, by definition, $\phi<^{\prime} \psi$ iff $\phi \wedge \psi<\psi$ and $\phi \wedge \psi \nless \phi$. Similarly, $\phi<" \psi \psi$ iff $\phi \wedge \psi<(\phi \wedge \psi) \vee \psi$ and $\phi \wedge \psi \nless(\phi \wedge \psi) \vee \phi$. Both of these conditions reduce to $\phi<\psi$, by Observation 5 and (EE1) - (EE3'). Q.E.D.

This shows that the bridge principles of Gärdenfors and Makinson get the transitions quite right, provided that both the contraction operation and the entrenchment relation are determined by a fixed belief base. The agreement is perfect in the case of entrenchment relations and only approximate in the case of contraction operations. Parts (c) and (d) to some extent reproduce the nice results of Gärdenfors and Makinson [9, Corollary 6].

However, it is hard to make intuitive sense of the occurrences of

${ }^{18}$ For any two contraction functions $\dot{-}_{1}$ and $\dot{-}_{2}$ over $K, " \dot{-}_{1} \subseteq{ }_{2}$," is of course short for " $K \dot{-}_{1} \phi \subseteq K \dot{-}_{2} \phi$ for every $\phi$ ". 
' $A$ ' and ' $V$ ' in the above bridge principles. This is why I suggest a more transparent way to think of the interdefinability between epistemic entrenchment and belief change.

$$
\begin{aligned}
\phi<\psi \text { iff } \psi \in K \dot{-}\langle\phi, \psi\rangle \text { and } \phi \notin K \dot{-}\langle\phi, \psi\rangle \text { (Definition 8) } \\
\text { Meet base specialization: if }- \text { is the meet contraction de- } \\
\text { termined by a prioritized base } H \text {, then, by Observations } 4 \\
\text { and } 6, \phi<\psi \text { is definable by } H \Delta \psi \ll H \Delta \phi \\
\text { Singleton reformulation: in so far as } K \dot{-}\langle\phi, \psi\rangle \text { is identical } \\
\text { with } K \dot{-}(\phi \wedge \psi), \phi<\psi \text { if and only if } \psi \in K-(\phi \wedge \psi) \text { and } \\
\phi \notin K \dot{ }(\phi \wedge \psi)
\end{aligned}
$$

$$
\psi \in K \dot{-} \phi \text { iff } y \in K \text {, and }[\phi] \ll[\phi, \psi] \text { or } \vdash \phi \text { (Definition 10) }
$$

Meet base specialization: if $\ll$ is the bunch entrenchment generated by a prioritized base $H$, then, by Observation 3 , meet contraction determined by $H$ coincides with EEEcontraction, and $\psi \in K \dot{-} \phi$ is definable by $H \Delta[\phi, \psi] \ll$ $H \Delta[\phi]$

Singleton interpolation: in so far as $\phi<\psi$ implies $[\phi] \ll$ $[\phi, \psi]$, and this in turn implies $\phi<\phi \vee \psi$, large and small EE-contractions can serve as upper and lower bounds of EEE-contractions (Observation 9)

Our deviation from the standard account is clear. We invoke sets with two elements as arguments for contraction operations and entrencinment relations. More specifically, we replace, in the direction from belief change to epistemic entrenchment, the contractions with respect to conjunctions by pick contractions, and in the direction from epistemic entrenchment to belief change, the entrenchments of disjunctions by bunch entrenchments.

What is the reward for this exercise? First and foremost, we get a better understanding of the relevant interrelations. They sometimes happen to reduce to the standard definitions. But what is really meant by the latter is, I submit, precisely what is made explicit by the new definitions. In one direction, I should think there is virtually no difference: $K-(\phi \wedge \psi)$ appears to be intuitively 
indistinguishable from $K \dot{-}\langle\phi, \psi\rangle$. In the other direction, however, it is only the restricted context of theory change by singletons that makes our new definition reduce to the old one: $[\phi] \ll[\phi, \psi]$ mayand must! - then be identified with $\phi<\phi \vee \psi$.

Secondly, we manage without reference to any particular connective of the object language. Thus the theory of epistemic entrenchment becomes applicable to systems using a severely restricted language. For instance, we can speak of the entrenchment of the nodes in inheritance nets or reason maintenance systems (also called "truth maintenance systems"). There ought to be a corresponding connective-free formulation of the so-called Gärdenfors postulates for contraction operations [7, Section 3.4]. The obvious suggestion is to replace occurrences of ' $K \dot{-}(\phi \wedge \psi)$ ' by ' $K \dot{-}\langle\phi, \psi\rangle$ '. The elimination of connectives, however, works only for belief contractions. Belief revisions constructed according to the Levi identity make use of negations, and there does not seem to be a straightforward way to avoid this.

At last, we should like to give two warnings. The connectivefree formulation of the theory of epistemic entrenchment relations and theory contractions is only a by-product of this paper, slightly improving on the presentation in [27]. It is not necessary for the analysis of syntax-based belief change which turns essentially on the syntactical structure of the items in a belief base. There is no immediate transfer of insights from belief base update to updates in inheritance networks or reason maintenance systems (RMSs) with their unstructured "nodes". It may be expedient for some purposes to identify RMS "justifications" with Horn clauses. But this certainly does not suffice for nonmonotonic systems. Our $\mathrm{Cn}$ is supposed to be monotonic.

Multiple contraction and extended epistemic entrenchment have been found to be an appropriate means for analyzing base contraction. However - and this is the second warning-, the concepts of multiple contraction and extended epistemic entrenchment themselves, cut loose from the special context of contraction functions determined by prioritized belief bases, are still very much in need of a thoroughgoing analysis. This is evidently beyond the scope of the present paper. 


\section{References}

[1] Alchourrón, Carlos, and David Makinson (1982): "On the logic of theory change: Contraction functions and their associated revision functions", Theoria 48, 14-37.

[2] Alchourrón, Carlos, and David Makinson (1985): "On the logic of theory change: Safe contraction", Studia Logica 44, 405-422.

[3] Alchourrón, Carlos, Peter Gärdenfors and David Makinson (1985): "On the logic of theory change: Partial meet contraction and revision functions", Journal of Symbolic Logic 50, 510-530.

[4] Dubors, Didier, and Henri Prade (1991): "Epistemic entrenchment and possibilistic logic", Artificial Intelligence 50, 223-239.

[5] Fuhrmann, André (1988): Relevant Logics, Modal Logics, and Theory Change, PhD thesis, Australian National University, Canberra.

[6] Fuhrmann, André (1991): "Theory contraction through base contraction", Journal of Philosophical Logic 20, 175-203.

[7] Gärdenfors, Peter (1988): Knowledge in Flux: Modeling the Dynamics of Epistemic States, Bradford Books, MIT Press, Cambridge, Mass.

[8] Gärdenfors, Peter (1990): "The dynamics of belief systems: Foundations vs. coherence theories", Revue Internationale de Philosophie 44, $24-46$.

[9] Gäridenfors, Peter, and David Makinson (1988): "Revisions of knowlcdge systems using epistemic entrenchment", in Moshe Vardi ed., Proceedings of the Second Conference on Theoretical Aspects of Reasoning about Knowledge, Morgan Kaufmann, Los Altos, Ca., pp. 83-95.

[10] Hansson, Sven Ove (1989): "New operators for theory change", Theoria 55, 114-132.

[11] Hansson, Sven Ove (1993a): "Reversing the Levi identity", Journal of Philosophical Logic 22, 637--669.

[12] HansSON, Sven Ove (1993b): "Theory contraction and base contraction unified", Journal of Symbolic Logic 58, 602-625.

[13] Kratzer, Angelika (1981): "Partition and revision: The semantics of counterfactuals", Journal of Philosophical Logic 10, 201-216.

[14] Harman, Gilbert (1986): Change in View, Bradford Books, MIT Press, Cambrige, Mass.

[15] Lrvesque, Hector J. (1984): "A logic of implicit and explicit belief", Proceedings 3rd National Conference on Artificial Intelligence, Austin TX, pp. 198-202.

[16] Lindström, Sten, and Wlodzimierz RABinowicz (1991): "Epistemic entrenchment with incomparabilities and rclational belief revision", in André FunRmann and Michael Morriau eds., The Logic of Theory Change, Springer, Berlin, pp. 93-126.

[17] Makinson, David (1985): "How to give it up: A survey of some formal 
aspects of the logic of theory change", Synthese 62, 347-363.

[18] Makinson, David (1993): "Five faces of minimality", Studia Logica 52, 339-379.

[19] MCARTHUR, Gregory L. (1988): "Reasoning about knowledge and belief: A survey", Computational Intelligence 4, 223-243.

[20] NeBel, Bernhard (1989): "A knowledge level analysis of belief revision", in Ronald Brachman, Hector Levesque and Raymond Rister eds., Principles of Knowledge Representation and Reasoning. Proceedings of the Ist International Conference, Morgan Kaufmann, San Mateo, Ca., pp. 301-311.

[21] NeBel, Bernhard (1992): "Syntax-based approaches to belief revision", in Peter Gärdenfors ed., Belief Revision, Cambridge University Press, Cambridge, pp. 52-88.

[22] Poole, David (1988): "A logical framework for default reasoning", Artificial Intelligence 36, 27-47.

[23] ResCHER, Nicholas (1964): Hypothetical Reasoning, North-Holland, Amsterdam.

[24] Rescher, Nicholas (1976): Plausible Reasoning, van Gorcum, Assen.

[25] Rotr, Hans (1991): "Two methods of constructing contractions and revisions of knowledge systems", Journal of Philosophical Logic 20, 149 173.

[26] Rotr, Hans (1992a): "On the logic of theory change: More maps between different kinds of contraction function", in Peter Gärdinfors ed., Belief Revision, Cambridge University Press, Cambridge, pp. 122141.

[27] Rotr, Hans (1992b): "Preferential belief change using generalized epistemic entrenchment", Journal of Logic, Language and Information 1, 45-78.

[28] RotT, Hans (1993): "Belief contraction in the context of the gencral theory of rational choice", Journal of Symbolic Logic 58, pp. 1426-1450.

[29] Veltman, F. (1976): "Prejudices, presuppositions and the theory of counterfactuals", in Jeroen GroenENDIJK and Martin STOKHor eds., Amsterdam Papers of Formal Grammar, Vol. I, Centrale Interfaculteit, Universiteit Amsterdam, pp. 248-281.

[30] Weydert, E. (1992): "Relevance and Revision: About Generalizing Syntax-based Belief Revision", in D. Plearce and G. Wagni:r eds., Proceedings JELIA '92, 3rd European Workshop on Logics in AI, Springer, Berlin, pp. 126-138. 